\title{
Corela
}

Cognition, représentation, langage

8-2 $\mid 2010$

Vol. $8, n^{\circ} 2$

\section{Un arbre sémasiologique pour la représentation d'un phylum étymologique}

La polysémie de fr. tourner, ang. to turn et it. tornare en contraste

Jacques François

\section{OpenEdition}

\section{Journals}

Édition électronique

URL : http://journals.openedition.org/corela/1744

DOI : $10.4000 /$ corela. 1744

ISSN : 1638-573X

Éditeur

Cercle linguistique du Centre et de l'Ouest - CerLICO

Référence électronique

Jacques François, "Un arbre sémasiologique pour la représentation d'un phylum étymologique », Corela [En ligne], 8-2 | 2010, mis en ligne le 27 octobre 2010, consulté le 02 mai 2019. URL : http:// journals.openedition.org/corela/1744 ; DOI : 10.4000/corela.1744

Ce document a été généré automatiquement le 2 mai 2019.

\section{c) (1)(2)}

Corela - cognition, représentation, langage est mis à disposition selon les termes de la licence Creative Commons Attribution - Pas d'Utilisation Commerciale - Partage dans les Mêmes Conditions 4.0 International. 


\title{
Un arbre sémasiologique pour la représentation d'un phylum étymologique
}

\author{
La polysémie de fr. tourner, ang. to turn et it. tornare en contraste
}

\section{Jacques François}

1 Cet $\operatorname{article}^{1}$ a un objectif primaire d'ordre méthodologique et un objectif secondaire d'ordre empirique, l'un et l'autre étant indissolublement liés. Du point de vue de la méthodologie, il s'agit d'élucider les différences entre trois modes de représentation de la polysémie lexicale - c'est-à-dire, dans la terminologie classique de la sémantique lexicale, du CHAMP SÉmAsiologique d'un item lexical

2 (a) la GRILLE SÉMASIOLOGIQUe organisée à partir du classement des différents sens de l'item à l'aide d'un jeu de valeurs affectées à un jeu de dimensions sémantiques, donc en termes COMPONENTIELS (cf. \$1),

3 (b) la CARTE SÉMANTIQUE ${ }^{2}$ organisée sous la forme d'un réseau associatif entre des sens qui ne font pas l'objet d'une décomposition en traits distinctifs (cf. \$2), et

4 (c) l'ARBRE SÉMASIOLOGiQue à base componentielle obtenu à partir de la hiérarchisation des traits sémantiques retenus dans la grille sémasiologique (cf. \$3).

5 Le $\$ 4$ établit un cahier des charges, c'est-à-dire un inventaire des opérations constituant successivement les trois grilles des verbes tourner, to turn et tornare et élaborant un arbre sémasiologique général dont les arbres propres à chacun des trois verbes sont les émanations. Le $\$ 5$ décrit la constitution des trois grilles, le $\$ 6$ celle de l'arbre sémasiologique général et des trois arbres concernant chacun des trois verbes. Sur cette base je peux calculer le degré d'autonomie de chacun des trois champs sémasiologiques. Enfin le $\$ 7$ répertorie différents développements possibles de cette étude

6 Les grilles comme les cartes visent à comparer la colexicalisation ${ }^{3}$ de deux ou plusieurs items appartenant à deux langues $L \alpha$ vs. $L \beta$ (perspective conTRASTIVE, voire typologique) ou à deux états successifs d'une même $e^{4}$ langue $E_{i} L \alpha$ vs. $E_{j} L \alpha$ (perspective DiachroniQUE). 
L'illustration que je développe plus bas inclut les deux perspectives : en latin classique l'expression verbale d'un mouvement rotatif complet ou partiel (c'est-à-dire d'un changement ou d'une inversion de direction) est assurée par les verbes gyrare, torquere, vertere, volvere qui ont connu des destins variés dans les langues romanes modernes ${ }^{5}$. En marge de ces verbes d'emploi général, le verbe latin tornare ne s'emploie que dans un sens artisanal, «fabriquer (une poterie) à l'aide d'un tour de potier ». Il va connaître une colexicalisation massive et différenciée en italien, en français et en anglais. Dans la mesure où l'angl. to turn est un emprunt au franco-normand tourner, l'écart entre les colexicalisations de ces deux items est faible, alors qu'il est important entre ceux-ci et l'italien qui réserve tornare à des emplois intransitifs (non causatifs) essentiellement d'inversion de direction ${ }^{6}$, tandis que le français et l'anglais emploient respectivement tourner et to turn dans des emplois transitifs et intransitifs avec une valeur +/-causative (verbes réversibles ou labiles) et pour désigner différents types de rotation, entière, partielle ou inversion de direction. Nous partirons de l'observation ${ }^{7}$ que le champ sémasiologique du verbe français est structurable grossièrement en fonction de quatre dimensions :

- la présence vs. absence d'un repère locatif complétée par

- (I') l'opposition entre une représentation spatiale et une représentation figurée (dimension conceptuelle),

- un aspect télique vs. atélique (dimension aspectuelle) et

- une valeur actancielle causative vs. non causative (dimension participative),

Dans la section 5, je décrirai plus finement la colexicalisation des trois verbes ${ }^{8}$ et je proposerai dans la section 6 une représentation de leur champ sémasiologique respectif sous forme d'un ARBRE SÉMASIOLOGIQUe À BASE COMPONENTIELLE. Avant cela il importe d'illustrer la différence entre une grille ou un arbre sémasiologique et une carte sémantique.

\section{Deux exemples de grille sémasiologique}

8 En introduction à son étude diachronique du concept « SE SOUVENIR »", Baldinger (1984) donne une représentation abstraite de la relation entre le signifié d'une " unité de base ", la variété des sémèmes qui lui est rattachée et un jeu de concepts organisés en sèmes susceptibles de distinguer ces sémèmes, cf. Figure 1. Baldinger représente conventionnellement ces traits distinctifs par une grille. 
Figure 1 : Représentation de l'articulation d'un signifié en sémèmes et de ceux-ci en 'concepts', c'est-à-dire en sèmes ou traits sémantiques distinctifs

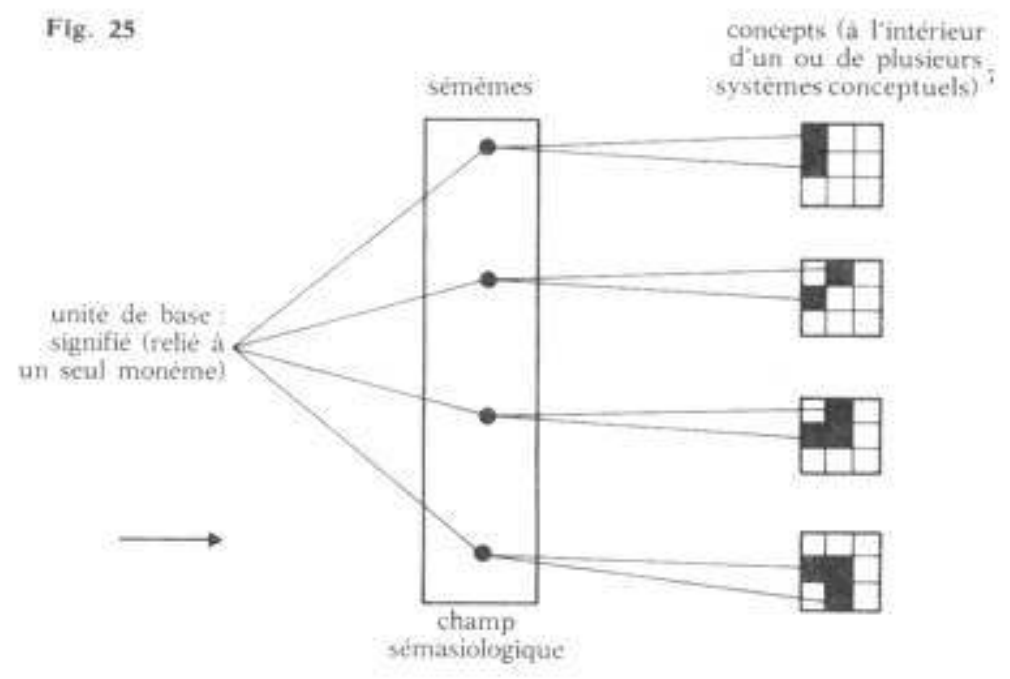

9 Supposons que cette grille (non explicitée par l'auteur) représente en colonnes un jeu de propriétés (A, B, C) et en lignes un jeu de valeurs $(1,2,3)$ dans chacune de ces propriétés : dans cette interprétation, le premier sémème serait constitué des sèmes $\{A 1 \text { ou } A 2\}^{10}$, le deuxième des sèmes $\{A 2$ et $B 1\}$, le troisième des sèmes $\{A 2$ et $\{B 1$ ou $B 2\}\}$ et le quatrième des sèmes $\{A 2$ et $\{B 2$ ou $B 3\}\}$. De ce fait les deux sémèmes les plus proches se révèlent être le $3^{\mathrm{e}}$ et le $4^{\mathrm{e}}$ car ils ont deux sèmes en commun $\{\mathrm{A} 2, \mathrm{~B} 2\}$.

On retrouve une idée apparentée dans l'étude de l'évolution de l'intension du signifié de l'item néerlandais legging (sorte de pantalon court féminin) proposée par D. Geeraerts dans son ouvrage sur la sémantique historique (1997:32-47). L'examen diachronique se base sur la recherche des traits distinctifs des illustrations de leggings dans des catalogues de mode durant 5 ans, de 1988 à 1992. Geeraerts distingue six « dimensions » descriptives et pour chaque dimension de deux à quatre valeurs. Les dimensions abrégées sont : $\mathrm{L}$ (ongueur), W(idth) (largeur vs. étroitesse), C(rease) (présence/absence d'un pli), M (atériau), $\mathrm{F}$ (onction) et $\mathrm{S}$ (exe).

11 Je modifie légèrement son mode de représentation en superposant les données des années 1988 et 1989. L'ordre des champs est: LWCMFS. La notation 11111f se lit:

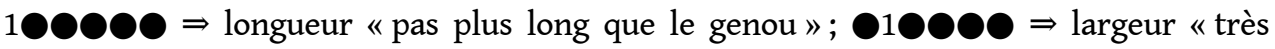
collant »; $01000=$ pli «absent »; $000100 \Rightarrow$ matériau « lisse, tissé ou tricoté finement "; 00010 fonction " porté comme un sur-vêtement, c'est-à-dire visible

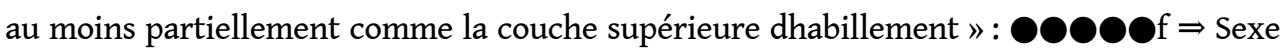
"féminin ». Les sémèmes barrés ne figurent qu'en 1988, ceux en italiques ne figurent qu'en 1989 et ceux en gras figurent dans les deux années. Le chiffre entre parenthèses indique le nombre d'illustrations du corpus correspondant à ce descriptif.

On constate entre 1988 et 1989 un noyau constant (3 sémèmes centraux) et pour le reste un glissement de sens avec la disparition de $\mathrm{L}=1$ (le legging s'arrête au-dessus du genou) et avec l'apparition de la configuration $\{2 / 3\} 1112 f$ (longueur jusqu'au dessous du genou ou jusqu'au-dessus de la cheville et fonction de sous-vêtement). Les combinaisons de valeurs sont représentées topologiquement dans des ensembles qui se superposent partiellement 
de manière à ce que chaque combinaison réalisée puisse figurer dans une case différente (les deux longueurs 2 et 3 étant regroupées en un seul ensemble), cf. Figure 2.

Figure 2 : Les six dimensions du champ sémasiologique de l'item néerl. legging entre 1988 et 1989 selon Geeraeerts (1997:36-37)

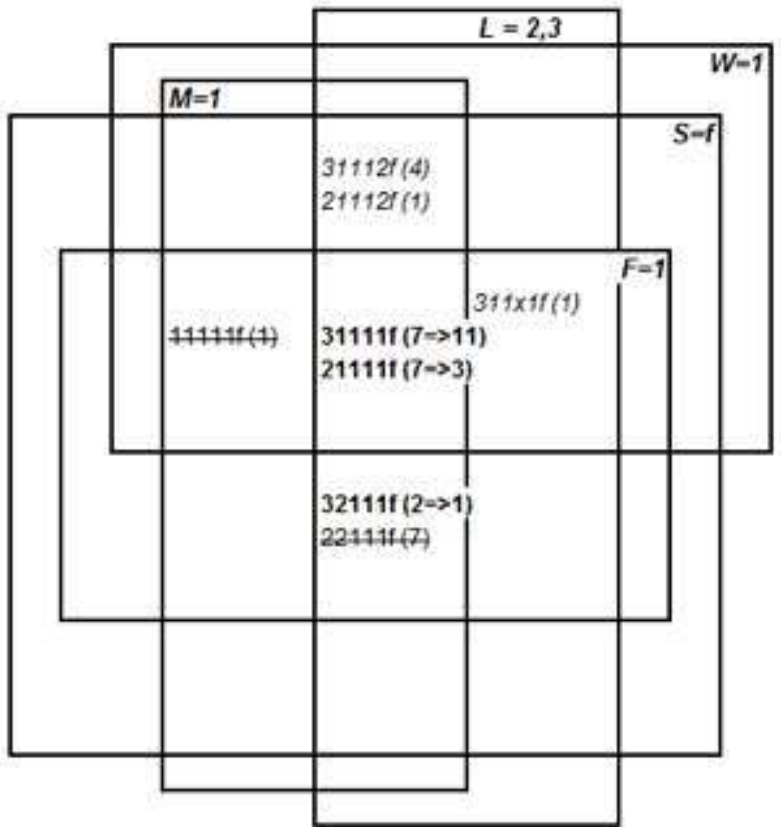

13 Ce type de représentation est relativement parlant à condition que le nombre de champs reste limité (les 6 champs représentés ici semblent constituer un maximum). En tout état de cause, il est explicitement componenTIEL, ce qui n'est pas le cas pour les deux types de cartes sémantiques que j'examine au \$2, et c'est une différence majeure.

\section{Deux exemples de carte sémantique}

Depuis plus d'un quart de siècle (cf. Anderson 1982, 1986) l'usage de cartes sémantiques s'est progressivement répandu en linguistique, plus particulièrement en typologie des langues. En septembre 2007, Michael Cysouw, Martin Haspelmath et Andrej Malchukov organisaient à l'Institut Max Planck d'anthropologie évolutionniste de Leipzig un atelier intitulé "Semantic Maps - Methods and Applications », dont l'argumentaire décrit ce qu'on attend généralement d'une carte sémantique en linguistique comparative :

In recent years the semantic map methodology has enjoyed increased popularity in cross-linguistic studies. Although there are various ways to make semantic maps, they all are attempts to visually represent cross-linguistical regularity in semantic structure. It has become increasingly clear that these attempts to map out linguistic categorisation provide an empirically testable tool to the study of semantic variation across languages. The semantic map approach has further shown convergence with grammaticalization theory, as well as with the research using (implicational) hierarchies, as found in functional typology and optimality theory. ${ }^{11}$

Une carte sémantique, telle qu'elle a été proposée par exemple par M. Haspelmath (2009) pour comparer les emplois du datif allemand et des prépositions à et to respectivement en français et en anglais ou par A. François (2008) comme outil de représentation de la polysémie nominale conçue comme la «colexicalisation ", dans des items de différentes 
langues, de différents sens (formant des «ensembles isolectaux ») ${ }^{12}$ en rapport plus ou moins étroit avec un noyau sémantique, diffère fondamentalement d'une grille à base componentielle

Un premier exemple assez élémentaire nous est fourni par Haspelmath (2009), l'un des trois organisateurs de l'atelier mentionné ci-dessus, qui défend ainsi la représentation d'un champ sémasiologique par une carte sémantique :

Pour les significations lexicales et grammaticales, la variabilité des langues ainsi que les ressemblances entre les langues se laissent représenter au mieux à l'aide de cartes sémantiques.(...) Cette carte montre correctement comment les différentes langues divisent la substance sémantique de manière diversifiée sans se recouvrir. (Haspelmath 2009 :19).

Il illustre cette méthode en comparant en termes de rôles sémantiques les différents emplois du datif de l'allemand, de la préposition à en français et de la préposition to en anglais (figure 3).

Figure 3 : Carte sémantique de marqueurs similaires au 'datif' et distribution du français à, de I'anglais to et du datif allemand selon Haspelmath $(2009: 20)$

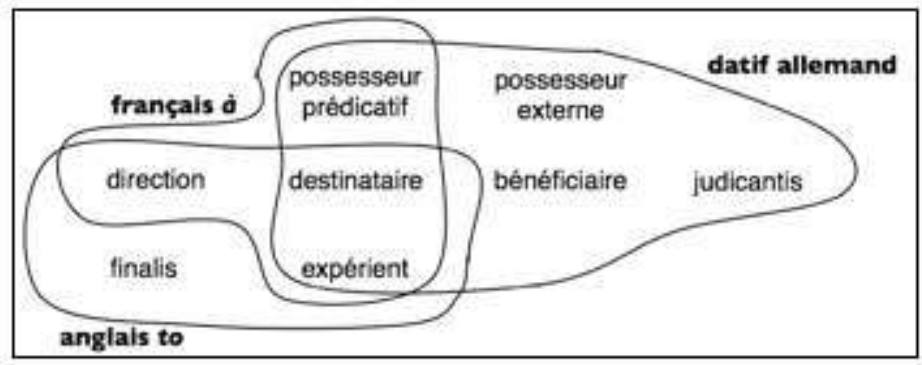

La représentation sous forme de grille serait la suivante (Tableau 21). Sous l'une ou l'autre forme, on voit clairement que le datif de l'allemand présente un champ sémasiologique plus diversifié ( 7 rôles) que celui des deux prépositions (4 rôles chacune) et qu'il partage trois rôles (le destinataire, la direction et l'expérient) avec les deux propositions. En outre ces prépositions ne partagent aucun autre rôle.

Tableau 1 : Essai de représentation de la carte sémantique de Haspelmath (2009) sous forme de grille (non componentielle)

\begin{tabular}{|l|c|c|c|}
\cline { 2 - 4 } \multicolumn{1}{c|}{} & alld datif & fr. $\dot{\boldsymbol{a}}$ & angl. to \\
\hline bénéfici aire & 1 & 0 & 0 \\
destinataire & 1 & 1 & 1 \\
direction & 1 & 1 & 1 \\
expérient & 1 & 1 & 1 \\
'finalis' & 0 & 0 & 1 \\
'judicantis' & 1 & 0 & 0 \\
possesseur exteme & 1 & 0 & 0 \\
possesseur prédicatif & 1 & 1 & 0 \\
\hline Total & 7 & 4 & 4 \\
\hline
\end{tabular}

À ce niveau élémentaire, on ne peut donc pas dire que la carte sémantique offre un avantage représentationnel évident face à la grille sémasiologique. Dans aucun des deux cas il n'y a d'analyse componentielle, c'est-à-dire que les rôles ne sont pas décomposés en traits comme cela a pu être fait, par exemple par Nilsen (1972) dans les débuts des grammaires de cas sémantiques et ultérieurement par Dowty (1991) dans le cadre de sa théorie des rôles prototypiques ou par van Valin (2005) dans le cadre de sa théorie des rôles hiérarchisés. 

propose une grille sémasiologique (elle aussi non componentielle) de la colexicalisation d'un vaste échantillonage d'items de différentes langues actuelles et anciennes en rapport avec le concept de RESPIRATION (Tableau 2 en Annexe).

21 Sur cette base, il construit une carte sémantique généralisée, c'est-à-dire incluant par association tous les sens colexicalisés dans le signifié d'au moins l'un de ces items (Figure 4).

Figure 4 : Carte sémantique généralisée des associations de sens autour du concept de RESPIRATION (A. François $2008: 202$ )

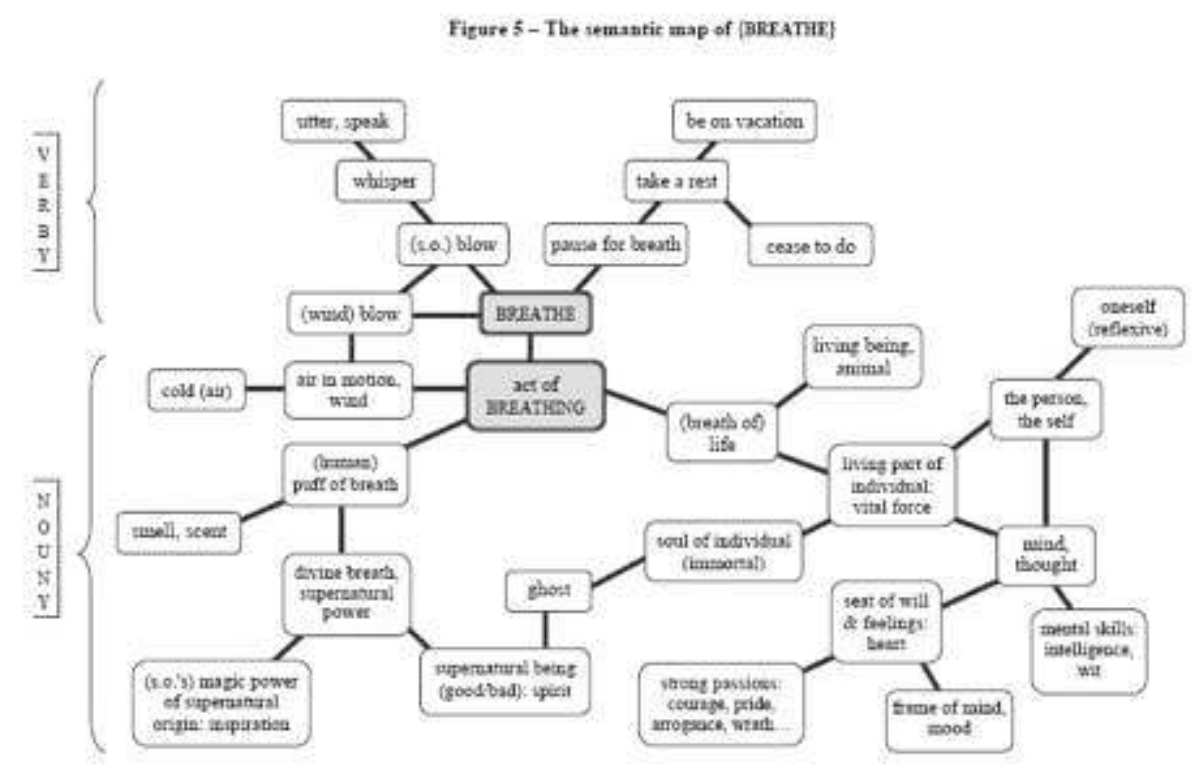

Sur cette double base (la grille et sa visualisation sous forme de carte sémantique), A. François associe à chacun des items comparés un "ensemble isolectal ", c'est-à-dire un sous-ensemble de la carte générale. Je me limiterai à la comparaison entre deux items du grec classique sémantiquement apparentés, pneuma et psūkhē. Dans la colexicalisation du gr. pneuma (figure 5) on retrouve une partie seulement des concepts associés dans la figure 4.

Figure 5 : Ensemble isolectal (simplifié) de gr. pneuma

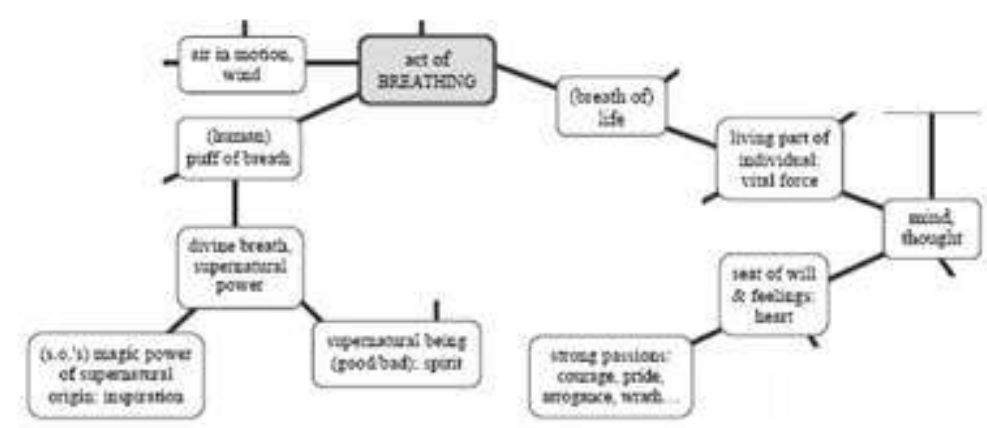

Et dans celle du gr. psūkhē (figure 6) on trouve également une partie de ces concepts, les deux ensembles isolectaux présentant une intersection. 
Figure 6 : Ensemble isolectal (simplifié) de gr. psūkhē

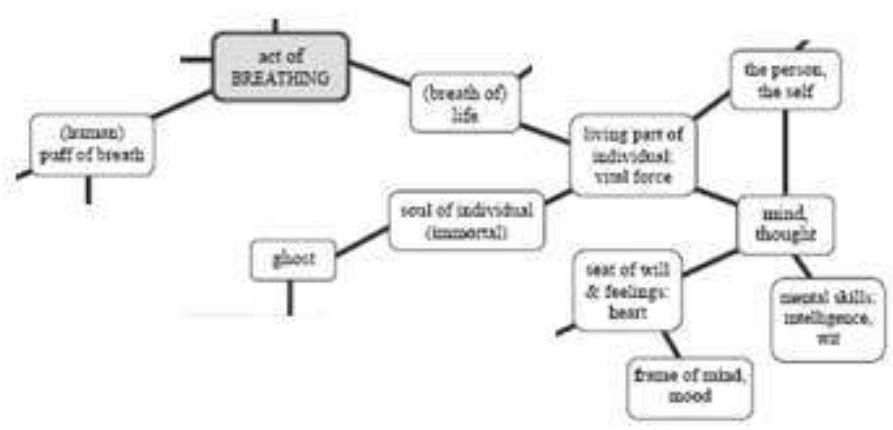

Le tableau 3 classe les concepts de la grille sémasiologique d'A. François selon qu'ils figurent dans les deux ensembles isolectaux de pneuma et psūkhe, dans un seul ou dans aucun des deux. Il est à noter que ces deux items n'ont que des emplois nominaux, ce qui exclut par principe toutes les acceptions de la partie supérieure de la carte générale, classées 'verby'.

Tableau 3 : Concepts associés dans la colexicalisation de gr. pneuma et gr. psūkhē

\begin{tabular}{|c|c|}
\hline concepts partagés & $\begin{array}{l}\text { acte de respiration ; (souffle de) vie ; force vitale ; souffle de respiration ; esprit, } \\
\text { pensée ; siège de la volonté et des sentiments, cceur }\end{array}$ \\
\hline $\begin{array}{l}\text { concepts } \\
\text { particuliers à gr. } \\
\text { pneuma }\end{array}$ & $\begin{array}{l}\text { air en mouvement ; vent ; souffle divin, pouvoir sumaturel ; pouvoir magique d'origine } \\
\text { sumaturelle ; inspiration ; etre sumaturel, bon/mauvais esprit; fortes passions : courage, } \\
\text { orgueil, amogance, etc. }\end{array}$ \\
\hline $\begin{array}{l}\text { concepts } \\
\text { particuliers à gr. } \\
\text { psilkhe }\end{array}$ & âme d'un individu ; fantôme ; cadre de l'esprit, mentalité \\
\hline $\begin{array}{l}\text { concepts exclus des } \\
\text { deux ensembles } \\
\text { isolectaux }\end{array}$ & $\begin{array}{l}\text { respiration ; souffle du vent ; souffler (qn) ; souffler un mot; parler, exprimer ; faire un } \\
\text { pause respiratoire ; prendre du repos ; cesser de faire ; être en congé; être animé. } \\
\text { animal ; (le) soi ; froid (air); senteur, effluve }\end{array}$ \\
\hline
\end{tabular}

Sur cette base, il est ensuite possible par exemple de comparer la paire d'items grecs pneuma $\sim$ psūkhē au triplet d'items correspondants en latin : animus $\sim$ anima $\sim$ spiritus. Nul doute qu'un tel examen minutieux ne présente un intérêt majeur pour mesurer le degré de parallélisme entre les représentations de l'espace du spirituel (l'esprit étant vu comme un souffle) dans ces deux cultures de l'antiquité européenne.

\section{Un exemple d'arbre sémasiologique destiné à l'examen de la polysémie verbale}

Finalement le mode de représentation des champs sémasiologiques des trois verbes le plus adapté semble être un arbre de classement ontologique du type proposé par Stein (2005) dans son étude de lexicographie computationnelle du lexique verbal de l'italien. Pour le verbe tornare, le classement de Stein ${ }^{13}$ distingue 32 acceptions mettant en cause quatre grands domaines ontologiques: matériel, social, mental et relationnel. Dans le domaine matériel, tornare est concerné par les mouvements (5 acceptions), les événements (10 acceptions) et les 'affectations' (5), dans le domaine social, tornare est concerné par un sens d'ordre verbal, dans le domaine mental par des actions mentales (5), des états mentaux (1) et des événements mentaux (1). Enfin dans le domaine relationnel, trois acceptions sont rattachées à une propriété fonctionnelle et une à une propriété 
physique. Le tableau 5 reproduit les trois premiers rangs de l'arbre de classement ontologique.

Tableau 4 : L'arbre de classement ontologique du champ sémasiologique du verbe it. tornare selon A. Stein (3 premiers rangs et nombre d'acceptions regroupées)

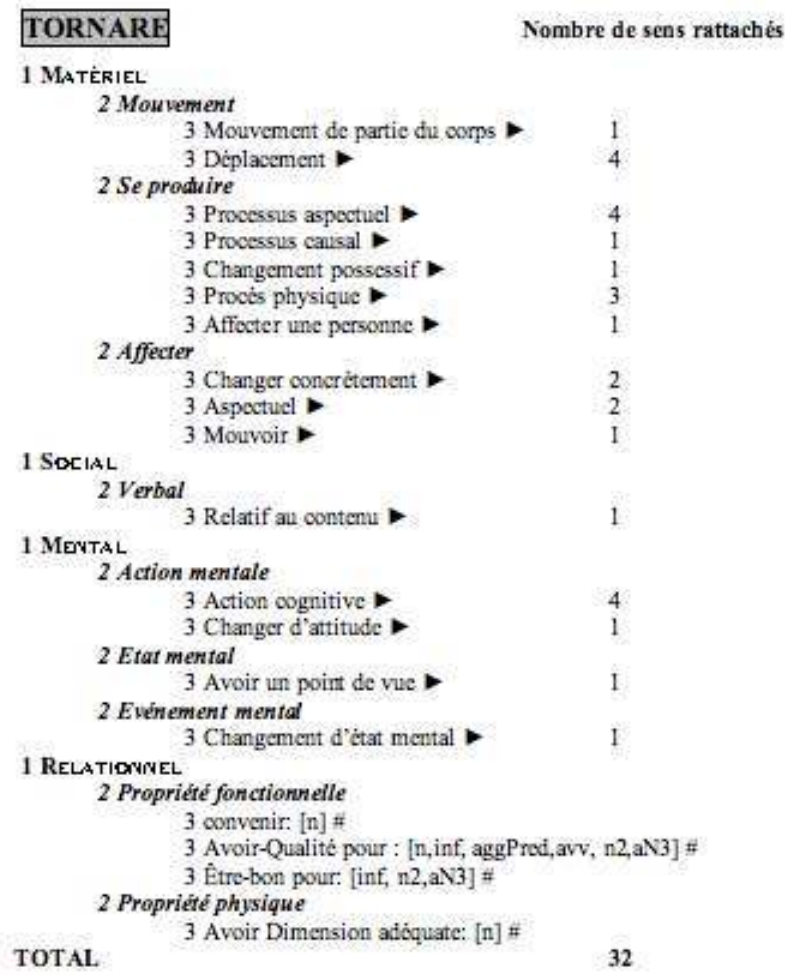

Deux différences principales sont à souligner entre l'arbre de classement ontologique d'A. Stein et la carte sémantique d'A. François :

a. A l'exception du centre de la carte occupé par un concept relativement vague, les positions de la carte sémantique sont occupées par des concepts structurés, tandis que les positions intermédiaires sur l'arbre de classement ontologique sont occupées par des traits élémentaires hiérarchisés, de sorte que seules les feuilles de l'arbre représentent les différentes acceptions décrites chacune par un cheminement différent sur l'arbre.

b. Les relations de proximité entre concepts structurés sont représentées directement sur la carte (proximité de niveau 1 entre deux pavés adjacents, de niveau 2 entre deux pavés séparés par un troisième, etc.), tandis que sur l'arbre elles correspondent au nombre d'arcs qu'il faut parcourir pour accéder d'une feuille à une autre. L'intérêt de ce type de représentation arborescente a été mis en évidence en particulier par L. Cavalli-Sforza dans sa mise en correspondance des familles linguistiques et des relations génétiques entre les peuples parlant les langues concernées ${ }^{14}$.

Ce type de représentation arborescente s'apparente à la grille sémasiologique de Geeraerts par leur base componentielle comparable. La différence majeure entre les deux représentations est que la grille sémasiologique de Geeraerts, constituée d'espaces qui se recouvrent partiellement, ne hiérarchise pas les 6 traits distinctifs (voir plus haut) alors qu'une représentation arborescente implique une hiérarchisation des traits, laquelle peut se révéler problématiqu ${ }^{15} \mathrm{si}$ l'on s'intéresse particulièrement au degré de proximité entre les acceptions. 
Dans la mesure où tel n'est pas mon propos (il s'agit ici de calculer le degré global de recouvrement des trois ensembles isolectaux, quelles que soient les relations entre les acceptions), j'opte pour ce mode de représentation qui permet de repérer aisément toutes les feuilles de l'arbre qui sont partagées par deux des verbes ou les trois et donc de calculer le degré de recouvrement entre les trois champs sémasiologiques.

\section{Questions préalables à l'élaboration de l'arbre sémasiologique général}

\section{Observations primaires sur la diversification des sens des 4 verbes}

Pour comparer la colexicalisation des verbes en examen, il faut disposer d'un tertium comparationis. Bien entendu la dimension conceptuelle et la dérivation figurative évoquées plus haut jouent un rôle essentiel, mais il ne faut pas négliger les propriétés aspectuelles (ou Aktionsart) et participatives du syntagme verbal. Parmi les propriétés aspectuelles (dynamicité, transitionalité, télicité) on constate que les trois verbes français anglais et italien présentent des emplois aussi bien atéliques que téliques, alors que le verbe source latin tornare exprime un seul type de mouvement hybride : atélique du point de vue de la rotation du tour de potier sur son axe, mais télique du point de vue de la poterie qui est progressivement façonnée.

C'est évidemment le destin du référent de l'objet à l'accusatif (la poterie) qui importe ici, donc la télicité, puisqu'une fois façonnée, la poterie atteint un état final (son telos). Du point de vue des propriétés participatives, c'est la causativité qui est le trait pertinent, car le verbe latin ne présente qu'un type d'emploi causatif (le façonnage de la poterie) alors que le verbe italien ne présente lui que des emplois non causatifs (l'expression causative implique l'adjonction de l'auxiliaire de diathèse causative fare), c'est un verbe 'inergatif' dans la terminologie de la grammaire relationnelle (cf. Blake 1991 :29sq).

En revanche les deux verbes français et anglais présentent une facette causative (sujet $\rightarrow$ agent $X$ / objet $\rightarrow$ patient $Y$ ) et une facette non causative (sujet $\rightarrow$ patient $Y$, pas d'objet). Ce sont des verbes ergatifs, réversibles, labiles ou anticausatifs, selon différentes terminologies. Le tableau 5 indique combien de sens des quatre verbes sont répertoriés dans des dictionnaires de référence ${ }^{16}$ et si l'on rencontre des types d'emploi téliques ou atéliques et causatifs ou non causatifs. 
Tableau 5 : La diversification des sens des 4 verbes selon la lexicographie (G, LVF, OED, WIV) et leurs parasynonymes

\begin{tabular}{|c|c|c|c|c|c|}
\hline & \multirow[t]{2}{*}{$\begin{array}{l}\text { eventail des } \\
\text { sens }\end{array}$} & \multirow[t]{2}{*}{$\begin{array}{l}\text { Aspect : } \\
\text { têlicité }\end{array}$} & \multirow[t]{2}{*}{$\begin{array}{l}\text { Participation : } \\
\text { causativité }\end{array}$} & \multicolumn{2}{|c|}{ Verbes concurrents } \\
\hline & & & & $\begin{array}{l}\text { sémantiquement apparentés et } \\
\text { morphologiquement } \\
\text { indépendants }\end{array}$ & $\begin{array}{l}\text { morph. apparentés } \\
\text { ct sém. } \\
\text { indépendants. }\end{array}$ \\
\hline $\begin{array}{l}\text { lat. } \\
\text { tornare }\end{array}$ & $\begin{array}{l}\text { G: } 1 \text { sens } \\
\text { \& fabriquer } \\
\text { à l'aide d'un } \\
\text { tour * }\end{array}$ & + tel & +caus & $\begin{array}{l}\text { girare; torquere; vertere; } \\
\text { solvere }\end{array}$ & $x_{1}$ \\
\hline fr. tourner & $L V F: 27$ sens & $+/$-tel & $+/$ - caus: & $\begin{array}{l}\text { Changer ; graviter; pivoter; } \\
\text { transformer ; virer ; ctc. }\end{array}$ & $\begin{array}{l}\text { contosirner: } \\
\text { detourner: } \\
\text { retostoner }\end{array}$ \\
\hline $\begin{array}{l}\text { angl. to } \\
\text { turn }\end{array}$ & $\begin{array}{l}\text { OED : } 74 \\
\text { sens }\end{array}$ & $+/-\mathrm{tel}$ & +1 - caus & $\begin{array}{l}\text { to revolve; rotate ; spin; stir; } \\
\text { toss; veer; work etc. }\end{array}$ & $\begin{array}{l}\text { return; furn }+ \\
\text { PARTICULE }\end{array}$ \\
\hline it. tornare & $\begin{array}{l}\text { WWV : } 15 \text { sens } \\
+ \\
1 \text { emploi } \\
\text { d'auxiliaire } \\
(I V, \text { Stein } \\
2005: 32 \\
\text { sens) }\end{array}$ & t/-tel & - caus & $\begin{array}{l}\text { corrispondere ; quadrare: } \\
\text { riapparire; ricomparire; } \\
\text { reiterarsi ; rientrare; rincasare : } \\
\text { rinnowarsi ; ridientare: } \\
\text { riperersi ; venire }\end{array}$ & ritornare \\
\hline
\end{tabular}

\section{Questionnement et méthode d'analyse contrastive}

La question à laquelle nous allons tenter de répondre peut se formuler ainsi : Est-il possible de mesurer comparativement

a. l'éventail des sens des 3 verbes en contraste (abstraction faite de l'unique sens du lat. tornare ) et

b. l'écart relatif entre leurs champs sémasiologiques ou, dans la terminologie d'A. François, leurs ensembles isolectaux?

Pour répondre à cette double question, j'ai établi un cahier des charges en cinq points consistant à

a. établir une liste hiérarchisée de dimensions susceptibles d'organiser l'analyse comparative incluant prioritairement les oppostions d'aspect et de participation ;

b. construire la grille sémasiologique des trois verbes fr. tourner, angl. to turn et ital. tornare;

c. construire un arbre sémantique général intégrant toutes les acceptions répertoriées des trois verbes ;

d. indiquer pour chaque feuille de l'arbre lequel ou lesquels des trois verbes sont disponibles ;

e. calculer sur cette base le degré de recouvrement entre les trois ensembles isolectaux.

\section{La hiérarchisation des dimensions sémantiques}

La normalisation comparative des définitions de chacune des acceptions des trois verbes dégage trois types de traits constitutifs : aspectuel, participatif et conceptuel. Deux des trois verbes (à l'exception de l'it. tornare) étant réversibles (voir plus haut), il est préférable de disposer la propriété participative de causativité en queue de la hiérarchie. Trois valeurs sont à distinguer : procès subi (par un Patient), procès causé (par un Agent à l'encontre d'un Patient) et procès réflexif (valeur moyenne qui n'implique nullement une syntaxe réfléchie). Le trait distinctif aspectuel est la télicité : le procès est soit télique (ex. tourner la page), soit atélique (ex. tourner en rond pendant des heures). Cette opposition est 
essentielle et peut donc être disposée en tête de la hiérarchie. Entre ces deux types de propriétés, l'une aspectuelle et l'autre participative, on peut se limiter à trois niveaux de propriétés conceptuelles, le premier niveau étant occupé par la distinction parmi les procès atéliques entre mouvements et situations et parmi les procès téliques entre mouvements et changements (non spatiaux).

\section{Les trois grilles sémasiologiques}

\section{Grille sémasiologique de fr. tourner}

27 acceptions répertoriées dans la base Les verbes français sont à classer. On constate une grande variété d'acceptions relevant du mouvement télique, qui entrent dans quatre catégories: les changements de direction, les mouvements d'évitement (tourner une difficulté, un obstacle), les mouvements productifs accompagnés d'un objet effectué (tourner une poterie, un film, un compliment) et les rotations (tourner une sauce, la poignée tourne).

Tableau 6 : Grille sémasiologique de fr. tourner

\begin{tabular}{|c|c|c|c|c|c|c|}
\hline Niv1 & Niv2 & Niv3 & Niv4 & Niv5 & & fr. tourner \\
\hline Aspoct & Concl & Conc2 & Conc3 & Partic & LVF & Contextes \\
\hline \multirow{8}{*}{$\frac{\frac{a}{2}}{\frac{2}{2}}$} & \multirow[t]{5}{*}{ mvt } & \multirow{2}{*}{\multicolumn{2}{|c|}{ circ }} & Réfl. & 12 & $\begin{array}{l}\text { On totorne autour de la table. Les danseurs totornent sur le } \\
\text { plateau. }\end{array}$ \\
\hline & & & & Subi & 13 & La Terre towne autosir du Soleit \\
\hline & & \multirow[t]{3}{*}{ ds esp } & & Rél. & 15,17 & $\begin{array}{l}\text { On towene dans le bureau en attendant; , Le représentant } \\
\text { tourne dans la région. }\end{array}$ \\
\hline & & & & Subi & 19 & La route tourne beaucoup dans cette montagne. \\
\hline & & & meta & Subi & 20 & La conversation tourne sur $P$, sur la situation \\
\hline & \multirow[t]{3}{*}{ sit } & altem & & Subi & 16 & Le médecin tourne avec un collègue pour la garde. \\
\hline & & \multirow[t]{2}{*}{ rot } & & Subi & ? & La terre tourne sur son axe \\
\hline & & & meta & Subi & 14 & Le motear tourne régulliêrement. L usine toùrne à plein. \\
\hline \multirow{15}{*}{$\frac{\underline{\sigma}}{\frac{0}{3}}$} & \multirow[t]{3}{*}{ chang } & état & & Subi & 21,22 & Cet enfant tourne mal : Le lait la mayonnaise a tourné. \\
\hline & & \multirow{2}{*}{\multicolumn{2}{|c|}{ subst }} & Cause & 10 & On tourne cette remarque en plaisanterie. \\
\hline & & & & Subi & 24,25 & La discussion tourne en nixe. Le temps tourne d la pluie. \\
\hline & \multirow[t]{12}{*}{ mit } & \multirow[t]{6}{*}{ ch-dir } & & Réfl. & $\begin{array}{c}18 \\
27(s) \\
\end{array}$ & $\begin{array}{l}\text { On totorne d gauche dans le boulevard. On se tourne vers } \\
P \text {, wers la fenétre. }\end{array}$ \\
\hline & & & & Causé & 3 & On tourne une arme vers $P$, le fauteail vers la fenêtre. \\
\hline & & & & Subi & 23 & Le vent toume. \\
\hline & & & invers & Causé & 4 & On tourne une feaille. On tourne les pages diun live. \\
\hline & & & meta & Réfl. & $26(5)$ & On se tourne vers d'autres études, vers d'augres solutions. \\
\hline & & & & Causé & 5,6 & $\begin{array}{l}\text { On tourne la colère contre } P \text {; On tourne ses pensées vers } \\
\text { l'avenir. }\end{array}$ \\
\hline & & \multirow[t]{2}{*}{ evit } & & Objloc & 11 & On tosirne les positions adverses. \\
\hline & & & meta & Objloc & 7,8 & $\begin{array}{l}\text { On tourne la difficulté, lobstacle : On tourne la loile } \\
\text { rejglement. }\end{array}$ \\
\hline & & \multirow[t]{2}{*}{ prod } & & Cause & $\%$ & On towne we poterie, un film \\
\hline & & & meta & Cansé & 9 & On tourne son compliment agréablement. \\
\hline & & \multirow{2}{*}{\multicolumn{2}{|c|}{ rot }} & Cansé & 2 & On rosirne la salade, we sauce. \\
\hline & & & & Subi & 1 & La poignée tourne: \\
\hline
\end{tabular}

\section{Grille sémasiologique d'angl. to turn}

37 Comme je l'ai déjà dit, le verbe to turn est un emprunt au franco-normand, si bien que les deux champs sémasiologiques sont relativement analogues. Cependant on constate que les acceptions atéliques sont plus rares et l'anglais a développé de nombreux emplois téliques de changement avec des changements qualitatifs, substantiels ou quantitatifs. 
Tableau 7 : Grille sémasiologique du verbe ang. to turn

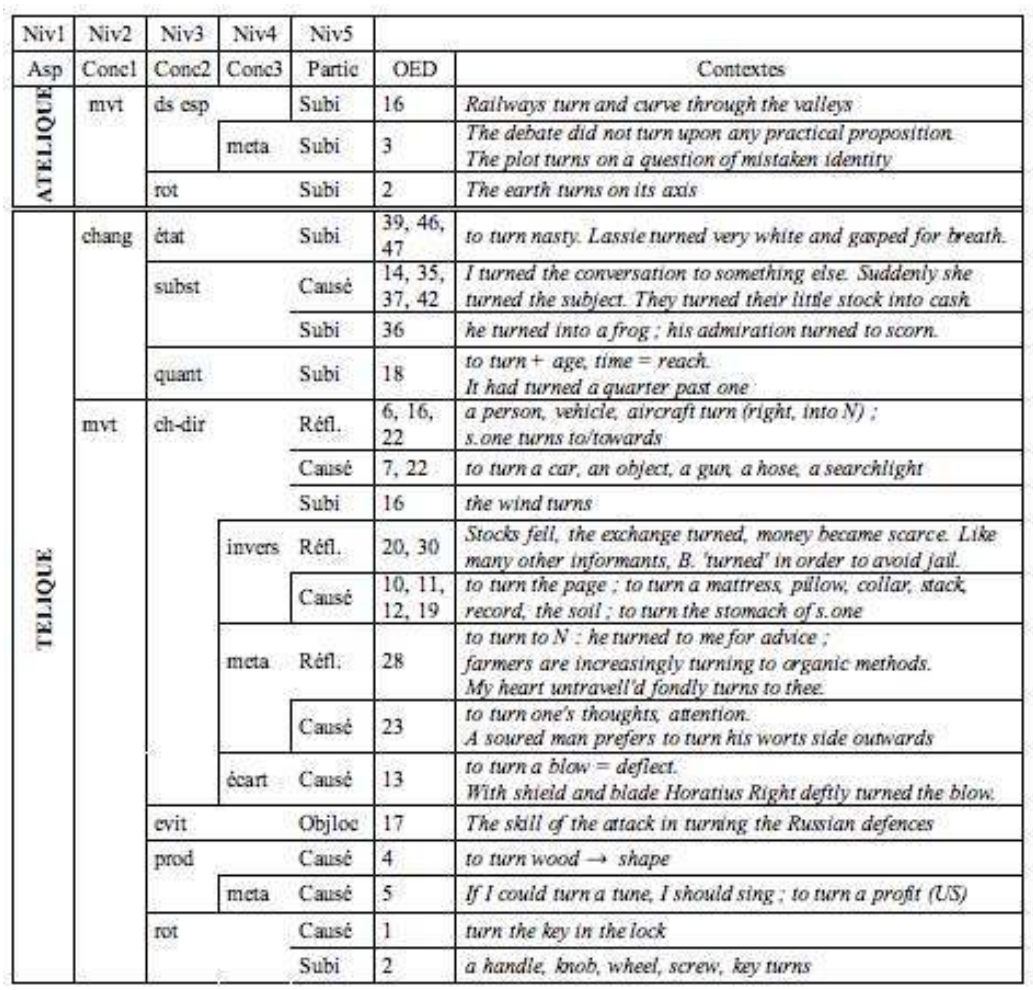

\section{Grille sémasiologique d'it. tornare}

Ici encore les acceptions atéliques sont minoritaires. Mais l'italien a développé des acceptions spécifiques, avec une variété de types de changements d'état et de changements de direction et dans les deux cas des valeurs de répétitition étrangères aux ensembles isolectaux de tourner et to turn. En outre l'italien colexicalise un emploi de semi-auxiliaire requérant une construction infinitive introduite par la préposition $a$. 
Tableau 8 : Grille sémasiologique du verbe it. Tornare

\begin{tabular}{|c|c|c|c|c|c|c|}
\hline \multirow{2}{*}{$\frac{\text { Nivl }}{\text { Aspect }}$} & \multirow{2}{*}{\begin{tabular}{c|c} 
Niv2 \\
Concl
\end{tabular}} & \multirow{2}{*}{\begin{tabular}{|c|} 
Niv3 \\
Conc2
\end{tabular}} & \multirow{2}{*}{\begin{tabular}{|c|} 
Niv4 \\
Conc3
\end{tabular}} & \multirow{2}{*}{$\frac{\text { Niv5 }}{\text { Partic }}$} & \multicolumn{2}{|r|}{ it. tornare } \\
\hline & & & & & B\&R & Contextes \\
\hline \multirow{3}{*}{ 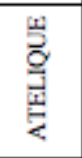 } & \multirow[t]{3}{*}{ sit } & \multirow[t]{2}{*}{ eval } & & Subi & 11 & $\begin{array}{l}\text { E son ragionamento che non torna. } \\
\text { Il two discorso non mi torna }\end{array}$ \\
\hline & & & rel & Subi & 12,15 & Mi torna meglo cosi Ti torna meglio associarti con isi \\
\hline & & \multicolumn{2}{|l|}{ qual } & Subi & 1 & Ciò che dici mi torna nuovo \\
\hline \multirow{11}{*}{ 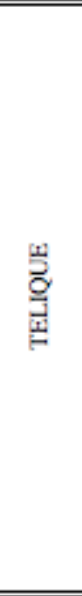 } & \multirow[t]{6}{*}{ chang } & \multirow[t]{4}{*}{ ètat } & & Subi & $7(\mathrm{a})$ & tornare sotto controllo; all'antico; all'opera \\
\hline & & & & Réfl. & $7(\mathrm{~b})$ & tornare all'opera ; al lavoro \\
\hline & & & invers & Subi & 5 & La tua camicia è tornata come nuova \\
\hline & & & repet & Subi & 10 & $\begin{array}{l}\text { Sta tornando l'inverno. } \\
\dot{E} \text { sue cura che fa tornare l'appetito. }\end{array}$ \\
\hline & & \multicolumn{2}{|l|}{ poss } & Subi & 6 & $\begin{array}{l}\text { Scaduto il contratto d'affitto il fondo torna al } \\
\text { proprietario. }\end{array}$ \\
\hline & & quant & dimin & Subi & 3 & I finghi, seccati, tornano circa un quinto del loro peso \\
\hline & \multirow[t]{5}{*}{ mvt } & \multirow[t]{5}{*}{ ch-dir } & invers & Réfl. & $2(\mathrm{a}), 4$ & $\begin{array}{l}\dot{E} \text { tornato da Palermo. Torniamo indietro : ci siamo } \\
\text { allontanati troppo. }\end{array}$ \\
\hline & & & repet & Réfl. & $2(\mathrm{~b})$ & Quanto tempo dovrà passare prima che tornino fra noi ? \\
\hline & & & $\begin{array}{l}\text { repet- } \\
\text { meta }\end{array}$ & Réfl. & 9 & tornare su wn'idea \\
\hline & & & meta & Rèfl. & 8 & $\begin{array}{l}\text { Ma torniamo ai due argomenti all'ordine del giorno nella } \\
\text { risnione odierna }\end{array}$ \\
\hline & & & écart & Réfl. & 13 & tornare il visa lo sguardo \\
\hline AUX & & chang & repet & (Transp) & 14 & Tornerò presto a farti visita Il sole è tornato a risplendere \\
\hline
\end{tabular}

\section{L'arbre sémasiologique général et ses configurations particulières à chacun des trois verbes}

\section{Le tronc de l'arbre ${ }^{17}$}

Il est constitué d'une double racine : l'une prédicative pour l'expression de procès téliques et atéliques, et l'autre auxiliarisée avec une valeur de changement répété représentée seulement en italien).

Figure 7 : Le tronc de l'arbre sémasiologique général

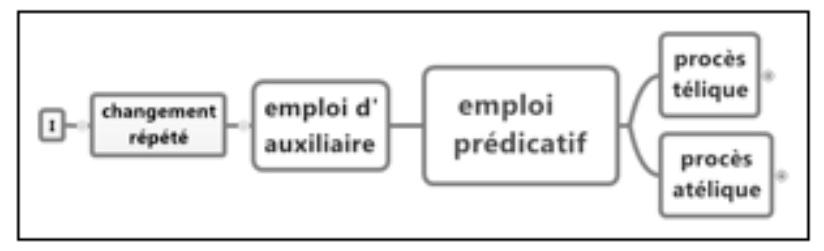

\section{Les branches maîtresses}

On distingue 8 classes intermédiaires de procès téliques, 4 pour les changements (non spatiaux) et quatre pour les mouvements, et 6 classes intermédiaires de procès atéliques, 2 pour les mouvements et 4 pour les situations. 
Figure 8 : Les branches maîtresses de l'arbre sémasiologique général

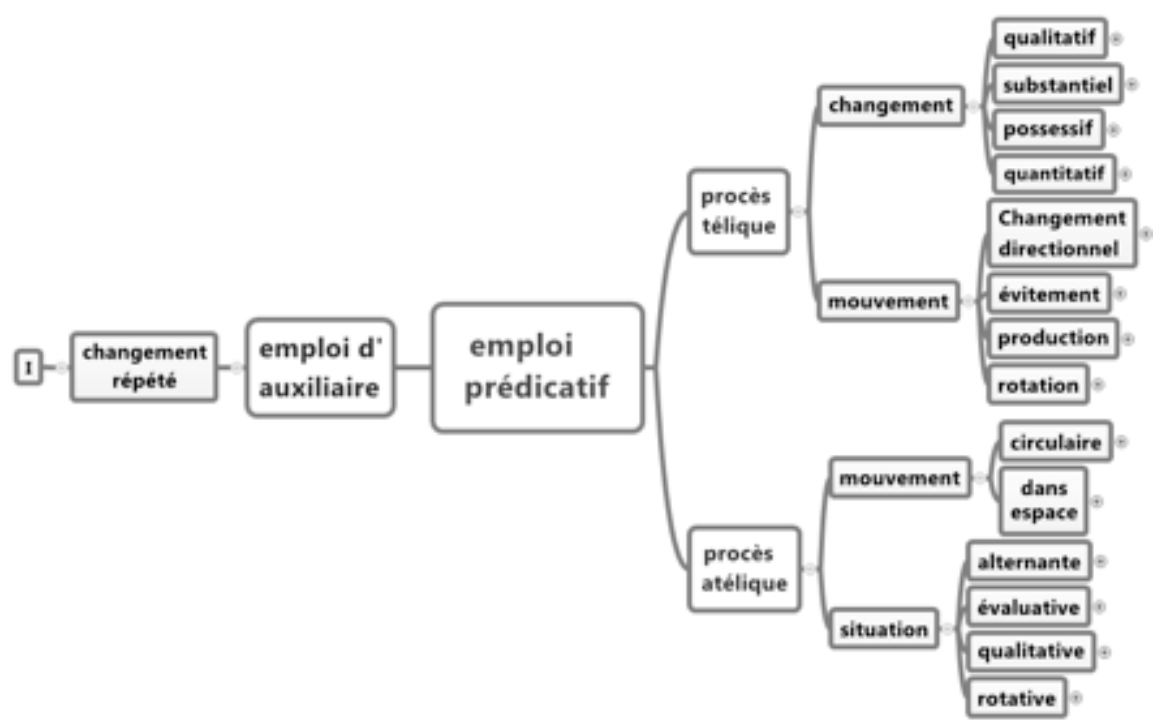

\section{L'arbre de colexicalisation de tourner (F, A-F, F-I, A-F-I)}

41 L'arbre sémasiologique général (c'est-à-dire l'union des arbres s'appliquant à chacun des trois verbes, non représenté ici faute de place) produit 38 feuilles. Sur ces 38 feuilles, 24 font partie du champ sémasiologique de tourner. Et sur ces 24 feuilles, 2 sont partagées par les trois verbes, 15 sont partagées avec to turn seul, une est partagée avec le seul tornare, et 6 sont propres au verbe tourner. Tourner a donc un faible degré d'autonomie, mais c'est avec to turn qu'il partage le plus d'acceptions (17 contre 3 avec tornare), comme on pouvait sy attendre, sachant que to turn (ainsi que to return) est un verbe emprunté au français. 5 des 6 acceptions spécifiques concernent l'expression de procès atéliques (cf. Figure 9). 
Figure 9 : L'arbre sémasiologique limité aux acceptions (partiellement partagées) de fr. tourner

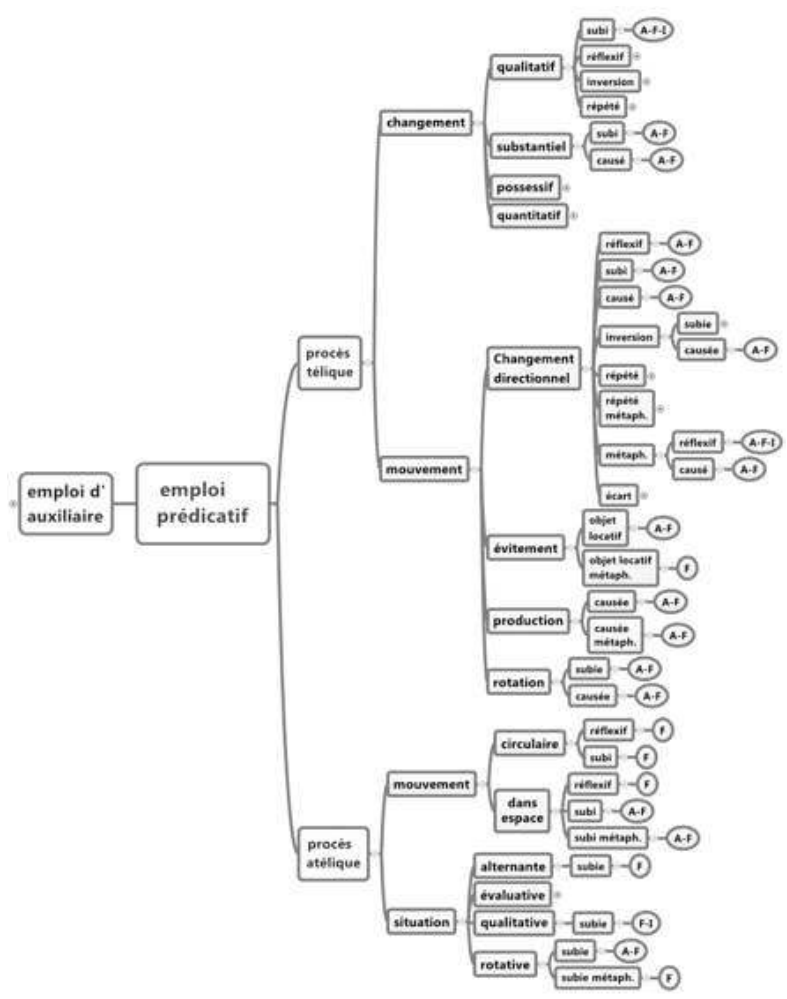

\section{L'arbre de colexicalisation de to turn (A, A-F, A-I, A-F-I)}

L'arbre limité aux acceptions de to turn en comporte 19 sur 38. En dehors des 2 acceptions partagées avec tourner et tornare et des 15 partagées uniquement avec tourner déjà repérées, on ne compte aucune acception partagée uniquement avec tornare, et seulement 2 acceptions spécifiques (cf. Figure 10). To turn présente donc un degré d'autonomie quasiment absolu par rapport à tornare, mais quasiment nul par rapport à tourner. 
Figure 10 : L'arbre sémasiologique limité aux acceptions (partiellement partagées) de to turn

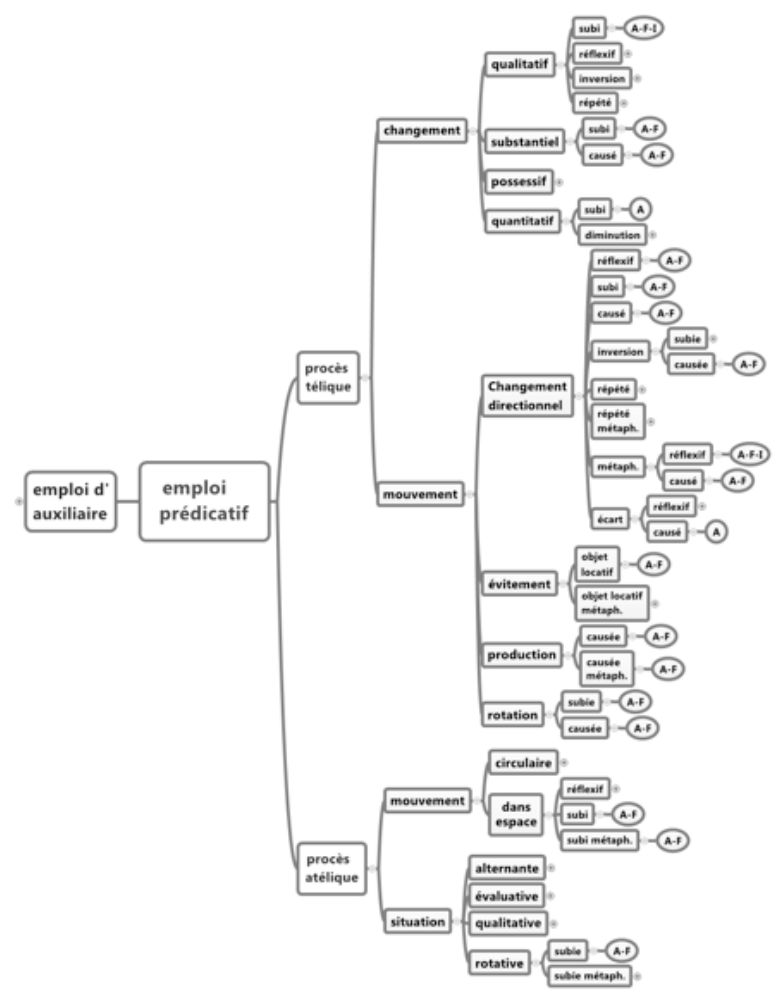

\section{L'arbre de colexicalisation de tornare (I, A-I, A-F-I)}

Enfin, l'arbre sémasiologique de tornare ne compte que 15 acceptions, se décomposant en deux acceptions partagées avec les deux autres verbes, une acception partagée seulement avec tourner, aucune avec to turn et 12 acceptions spécifiques (cf. Figure 11). Tornare a donc un degré d'autonomie élevé, ayant développé une quantité d'acceptions propres, mais jamais avec une valeur causative. 
Figure 11 : L'arbre sémasiologique limité aux acceptions (partiellement partagées) de tornare

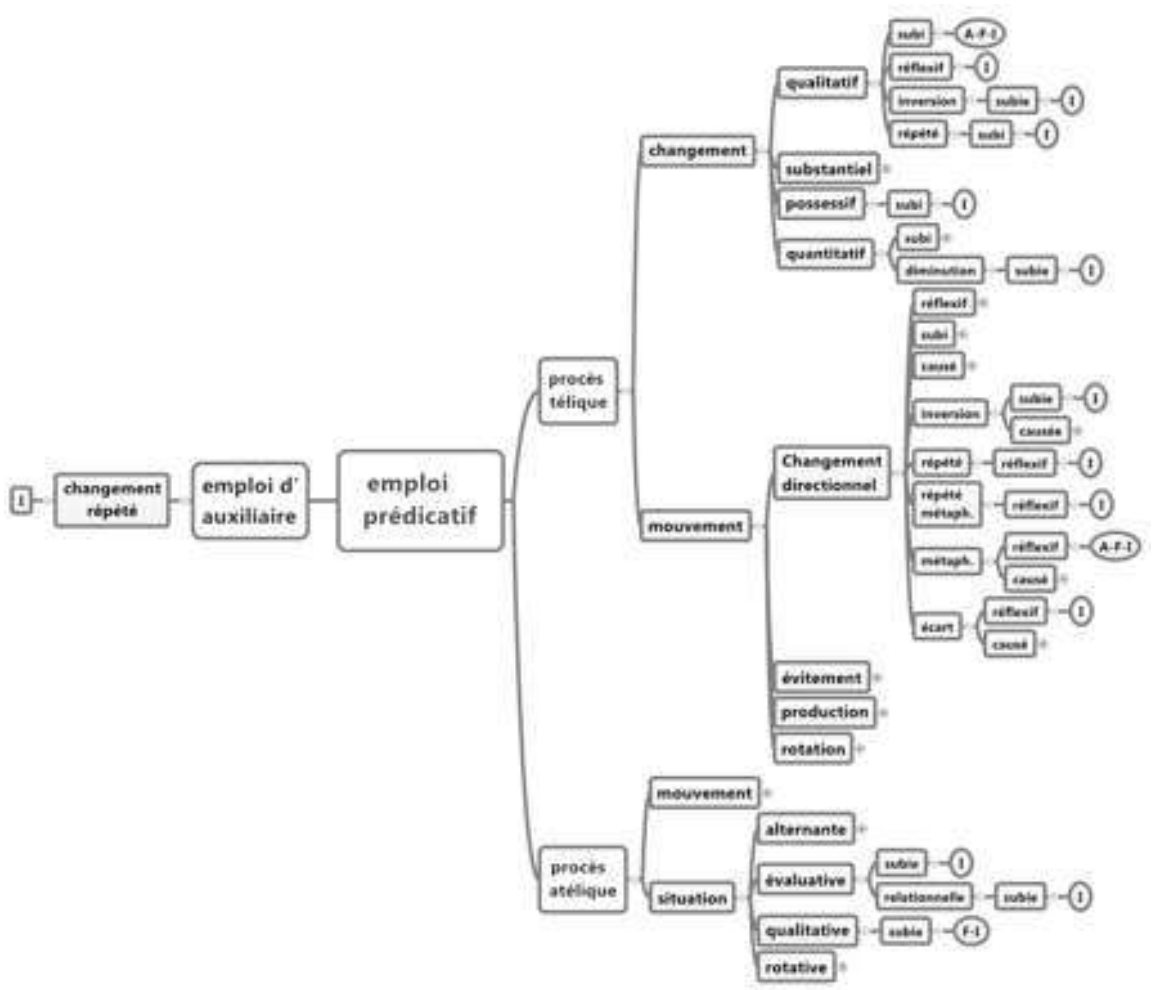

\section{Colexicalisation comparée des trois verbes}

On peut finalement représenter le recouvrement partiel des trois champs sémasiologiques représentés dans les figures 9-11 à l'aide d'une grille du même type que celles proposées par D. Geeraerts (cf. figure 2). Le champ de tourner partage deux acceptions avec ceux de to turn et tornare, une avec celui de tornare seul et 15 avec celui de to turn seul. Il comporte enfin six acceptions spécifiques. De son côté, le champ de to turn ne partage aucune acception avec tornare seul et a deux acceptions spécifiques. Quant au champ de tornare, il a 12 acceptions spécifiques. La figure 12 représente le recouvrement partiel de ces trois espaces sémasiologiques. Il permet de visualiser la forte autonomie de tornare (12 acceptions spécifiques sur 15, soit $80 \%$ ), l'autonomie médiocre de tourner ( 6 acceptions spécifiques sur 24 , soit $25 \%$ ) et enfin la faible autonomie de to turn (2 acceptions spécifiques sur 19, soit $\mathbf{1 1} \%$ ). Les deux acceptions partagées par les trois verbes sont

Procès télique $\boldsymbol{~ m o u v e m e n t} \boldsymbol{~ c h a n g e m e n t ~ d i r e c t i o n n e l ~} \boldsymbol{~ m e ́ t a p h o r i q u e ~} \boldsymbol{~ r e ́ f l e x i f , ~ e x . ~}$ 18

- fr. On se tourne vers d'autres études, vers d'autres solutions.

- angl. s.one turns to/towards N

- it. Ma torniamo ai due argomenti all'ordine del giorno nella riunione odierna

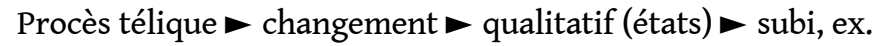

- fr. Cet enfant tourne mal ; Le lait,la mayonnaise a tourné .

- angl. Lassie turned very white and gasped for breath.

- it. tornare sotto controllo 
Figure 12 : Grille de partage des acceptions de tourner (F), to turn (A) et tornare (I)

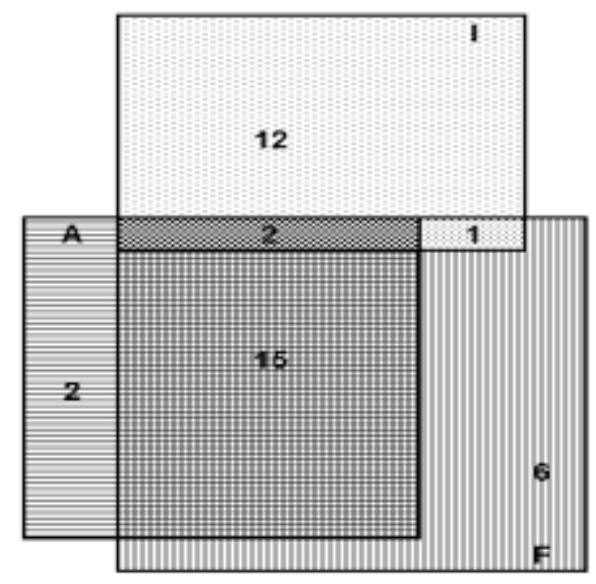

\section{Perspectives}

À l'aide d'un arbre sémasiologique général, c'est-à-dire distinguant les 38 acceptions spécifiques aux trois verbes tourner, to turn et tornare ou partagées par deux d'entre eux ou les trois, nous sommes parvenus à repérer les acceptions des trois verbes qui se recoupent (cf. figure 12). Au-delà de cette simple description contrastive, nous pouvons expliquer la faible autonomie relative des champs sémasiologiques de tourner et to turn par l'observation des conditions dans lesquelles to turn est né d'un emprunt au français ${ }^{19}$. En revanche tornare a suivi un cheminement étonnant (dont je n'ai pas l'explication) dans la mesure où ce verbe présente en italien moderne une variété d'emplois non causatifs et aucun emploi causatif, alors que le verbe source latin était monosémique et causatif (produire une poterie sur un tour de potier).

Cette étude peut être considérée comme le point de départ d'une recherche plus approfondie sur les points suivants :

- L'analyse devra être étendue aux composés anglais : to turn + particule et au dérivé to return

- La comparaison entre tourner et tornare suggère un développement pour tornare de la valeur d'inversion, exprimée en français et en italien par les dérivés retourner, ritornare et de celle d'écart exprimée en français par le dérivé détourner. Tourner est également occasionnellement synonyme de contourner (tourner les positions ennemies). Il faudra donc étendre la comparaison à tous les dérivés des 3 verbes, fr. contourner, détourner, retourner, angl. return, turn + ADV, it. ritornare.

- Par ailleurs il faudra compléter l'arbre sémasiologique général en recherchant les synonymes de chacun des 3 verbes susceptibles d'occuper les positions où tourner, to turn ou tornare n'est pas disponible. Cela concerne particulièrement toutes les constructions causatives, afin de voir si girare est apte à occuper toutes ces positions, comme le suggèrent les traductions proposées par les dictionnaires bilingues français-italien pour les emplois causatifs de tourner.

- Il faudra enfin examiner l'évolution historique comparée de tourner, to turn et tornare et des autres verbes exprimant un type de mouvement circulaire ou de changement de direction afin de comprendre comment les propriétés du sens de lat. tornare ne sont plus colexicalisées par le verbe italien et ne le sont plus que marginalement par tourner et to turn. 


\section{BIBLIOGRAPHIE}

Anderson, Lloyd B. (1982). The 'perfect' as a universal and as a language-particular category. In: Paul J. Hopper (ed.), Tense-Aspect: Between Semantics \& Pragmatics, 227-264. Amsterdam: Benjamins. Anderson, Lloyd B. (1986). Evidentials, paths of change, and mental maps: typologically regular asymmetries. In: Wallace Chafe \& Johanna Nichols (eds.) Evidentiality: The linguistic encoding of epistemology. Norwood: Ablex, 273-312.

Baldinger, K. (1966), Sémantique et structure conceptuelle (Le concept 'se souvenir'). Cahiers de lexicologie 8, p. 3-46.

Baldinger, K. (1984), Vers une sémantique moderne. Paris : Klincksieck

Blake, B. (1991), Relational Grammar. Londres: Routledge \& Kegan

Cavalli-Sforza, L. (1997), Des gènes, des peuples et des langues. Dossier Les langues du monde. Pour la Science, p. 42-49.

Dowty, D. (1991), Thematic proto-roles and argument selection. Language 67/3: 547-619.

François, A. (2008), Semantic maps and the typology of colexification - Intertwining polysemous networks across languages. In M. Vanhove (ed.), From polysemy to semantic change: towards a typology of lexical semantic. Amsterdam \& Philadelphie : Benjamins, p. 1-45

François, J. (1989), Changement, causation, action : trois catégories sémantiques fondamentales du lexique verbal français et allemand. Genève : Droz.

François, J. (1999), Les caractères aspectuels et participatifs des prédications verbales et la transitivité. BSL XCIV - Fascicule 1, p. 139-184.

François, J. (2003), La prédication verbale et les cadres prédicatifs. Louvain : Peeters.

François, J. (2008), Compte rendu de Stein (2005). BSL CIII - Fascicule 2, p. 58-66.

François, J. (2010), L'étude de la polysémie verbale entre dérivation et invariance. Conférence plénière au Congrès Mondial de Linguistique Française (CMLF2), La Nouvelle-Orléans [téléchargeable sur le site de l'Institut de Linguistique Française]

Geeraerts, D. (1997), Diachronic Prototype Semantics - A Contribution to Historical Lexicology. Oxford: Clarendon

Goldstein, K. (1931), Der Aufbau des Organismus. The Hague : Nijhoff.

Haspelmath, M. (2003), The geometry of grammatical meaning: semantic maps and crosslinguistic comparison. In M. Tomasello (ed.), The new psychology of language, Volume 2. New-York : Erlbaum, p. 211-243.

Haspelmath, M. (2009), Pourquoi la typologie des langues est-elle possible ? BSL CIV - Fascicule 1, p. 17-38.

Lazard, G. (2009), Pour une linguistique pure. BSL CIV - Fascicule 1, p. 1-16

Merleau-Ponty, M. (1942) La Structure du comportement. Paris : Presses Universitaires de France.

Nespoulous, J.L. (2005), La désintégration du langage dans le cerveau lésé de l'aphasique. In : J.M. Hombert (dir.), Aux origines des langues et du langage. Paris : Fayard, p. 428-449. 
Nilsen, D.L.F. (1972), English adverbials. The Hague : Mouton

Paulin, C. (à paraître), Du mouvement au changement d'état : étude de l'unité verbale turn. Faits de langue 2010.

Stein, A. (2005), Semantische Repräsentation italienischer Verben - Automatische Disambiguierung mit Konzepthierarchien. Tübingen : Niemeyer.

van der Auwera, J. \& Plungian, V.A. 1998. Modality's semantic map. Linguistic Typology, 2(1): 79-124.

van Valin, R. (2005), Exploring the Syntax-Semantics Interface. Cambridge University Press.

\section{Outils lexicographiques}

[G] Gaffiot, F, Dictionnaire latin-français [en ligne sur le site http://www.lexilogos.com/latin/ gaffiot]

[IV] Stein, A., Italienische Verben [base de données lexicales, cf. achim.stein@ling.unistuttgart.de]

[LVF] Dubois, J. \& F. Dubois-Charlier, Les verbes français [base de données lexicales en ligne, URL http://www.modyco.fr/?Ressources:Corpus_Dubois]

[OED] Oxford English Dictionary on historical principles (CD-ROM, 2002)

[WIV] Blumenthal, P. \& G. Rovere, Wörterbuch der italienischen Verben - Konstruktionen, Bedeutungen, Übersetzungen (1998)

\section{ANNEXES}

Tableau 3 : Grille sémasiologique multilingue dans l'espace de la RESPIRATION (A. François 2008 : 200-1)

\begin{tabular}{|c|c|c|c|c|c|c|c|c|c|c|c|c|c|c|c|}
\hline & $\begin{array}{l}\text { SANSRRAT } \\
\text { anmar }\end{array}$ & $\begin{array}{l}\text { Geser } \\
\text { psikhthe }\end{array}$ & $\begin{array}{c}\text { GFEEK } \\
\text { prewona }\end{array}$ & $\begin{array}{l}\text { LATIN } \\
\text { anima }\end{array}$ & $\begin{array}{c}\text { Lativ } \\
\text { spobithis }\end{array}$ & $\begin{array}{l}\text { RUSSIAN } \\
\text { dix }\end{array}$ & $\begin{array}{c}\text { VANOARN } \\
\text { qi }\end{array}$ & $\begin{array}{l}\text { REurt } \\
\text { anr- }\end{array}$ & $\begin{array}{l}\text { Naruati } \\
\text { imitho }\end{array}$ & $\begin{array}{l}\text { mothap } \\
\text { moökhe- }\end{array}$ & $\begin{array}{l}\text { Neanua } \\
\text { hored- }\end{array}$ & $\begin{array}{c}\text { AFbiC } \\
\text { nith }\end{array}$ & $\begin{array}{l}\text { Aaraic } \\
\text { nafas }\end{array}$ & $\begin{array}{l}\text { EEOAS } \\
\text { Silik }\end{array}$ & $\begin{array}{l}\text { SAR } \\
\text { koo }\end{array}$ \\
\hline BREATHE & {$[+]$} & {$[+]$} & {$[+]$} & & {$[+]$} & {$[+]$} & & {$[+]$} & & {$[+]$} & + & & {$[+]$} & & + \\
\hline (s.o) blow & & {$[+]$} & [t] & & {$[+]$} & {$[+]$} & & {$[+]$} & & {$[+]$} & + & & {$[+]$} & & + \\
\hline whisper, utter & & & & & & & & + & & & + & & & & \\
\hline take a rest & & & & & & [+] & & & + & {$[+]$} & + & {$[+]$} & {$[+]$} & & [+] \\
\hline be on vacation & & & & & & {$[+]$} & & & & {$[+]$} & & & & & \\
\hline cease to do & & & & & & & & & & & + & & & & \\
\hline (wind) blow & & & {$[+]$} & & {$[+]$} & {$[+]$} & & & & & & {$[+]$} & & & \\
\hline air, wind & {$[+]$} & {$[+]$} & + & + & + & {$[+]$} & + & & & & & {$[+]$} & & & + \\
\hline cold (air) & & {$[+]$} & & & & & & & & & & & & & \\
\hline puff of breath & + & + & + & + & + & + & + & + & + & + & + & + & + & + & + \\
\hline smell, scent & & & + & & + & {$[+]$} & + & & + & + & & {$[+]$} & & & \\
\hline ACT OF BREATHING & + & + & + & + & + & + & + & + & + & + & + & + & + & + & + \\
\hline (breath of iffe & + & + & + & + & + & + & + & + & & + & + & + & & & \\
\hline fiving being, animal & {$[+]$} & {$[+]$} & & {$[+]$} & & {$[+]$} & & & & & & & & & \\
\hline vital force of individual & + & + & + & + & + & + & + & + & & + & & + & + & + & \\
\hline person; self & + & + & & + & + & & & & & & & {$[+]$} & {$[+]$} & & \\
\hline oneself (reflexive) & + & & & & & & & & & & & {$[+]$} & {$[+]$} & & \\
\hline mind, thought & + & + & + & {$[+]$} & + & + & + & + & & & & + & {$[+]$} & & \\
\hline intelligence, wit & + & + & & & & & & & & & & & & & \\
\hline will and feelings: heart & & + & + & {$[+]$} & + & + & + & & & & & + & & & \\
\hline pride, arrogance, wrath. & & & + & {$[+]$} & + & + & {$[+]$} & & & & & & & & \\
\hline frame of mind, mood & & + & & {$[+]$} & + & + & + & & & & & \pm & & & \\
\hline soul of indiv. (immortal) & + & + & & + & + & {$[+]$} & & + & & & & + & & t & \\
\hline ghost & & + & & + & & + & & + & & & & + & & & \\
\hline divine breath or power & & & + & & + & + & & & & & & + & & & \\
\hline magic power, inspriation & & & + & & + & $1+1$ & & & & & & + & & & \\
\hline supematural being, dwinity & + & & + & & + & + & & & & & & + & & & \\
\hline
\end{tabular}




\section{NOTES}

1. Cet article est issu d'une communication dans la section Aspectualité - Temporalité Référentialité: les langues romanes en contraste $\mathrm{du} 31^{\mathrm{e}}$ Romanistentag (le congrès annuel des romanistes allemands) à Bonn le 28 septembre 2009. Je remercie Catherine Paulin de m'avoir encouragé à en publier les résultats, en marge de la parution prochaine de son propre article sur le verbe anglais to turn (2010), ainsi que deux relecteurs anonymes pour leurs observations attentives et critiques. Je retiendrai ici deux critiques de fond. La première concerne la corrélation entre la variation de sens et la variation de contexte. De mon point de vue c'est un faux problème, car c'est bien entendu la variation du contexte d'emploi qui révèle la variation de sens, quelle que soit la classe lexicale de l'item à l'étude. Concernant la sémantaxe des verbes, on distingue entre le contexte circonstanciel et le contexte régi (les arguments). Contrairement aux noms (à l'exception des noms d'action, d'événément, d'état ou de propriété) c'est ce contexte régi qui joue le rôle décisif dans la ventilation des sens et on a admis jusqu'à ces dernières années que c'est le verbe qui sélectionne ses arguments, mais récemment le pouvoir de sélection du prédicat par les arguments a été souligné (cf. François 2010). La seconde critique porte sur la hiérarchisation des propriétés adoptée pour la constitution de l'arbre sémasiologique général (et indirectement de chacun des arbres sémasiologiques des quatre verbes examinés). La préférence pour une prise en compte tardive de la causativité résulte de ma critique de son traitement précoce dans l'ontologie d'A. Stein (cf. François 2009). En effet dans le cas des verbes réversibles (ou anticausatifs), disposer l'opposition +/-causatif en tête de classement éloigne artificiellement des sens qui ne se distinguent que par la mention ou l'omission du rôle du causateur. Quant à la hiérarchisation de la propriété aspectuelle de télicité (intervenant ici en premier) et des propriétés conceptuelles (intervenant en second), elle peut effectivement être mise en doute, et il faut y voir essentiellement une commodité afin de dégager une structure arborescente.

2. On rencontre quatre désignations : carte sémantique, mentale, cognitive ou conceptuelle.

3. Ma traduction pour colexification proposé par A. François (2008).

4. Une comparaison de la colexicalisation de deux items appartenant au même état $E_{i}$ de deux langues $\mathrm{L} \alpha$ vs. $\mathrm{L} \beta$ n'est pertinente que si par «même état de langue " on entend des états correspondant à une même époque historique et s'il y a de bonnes raisons de supposer qu'à cette époque des deux langues $\mathrm{L} \alpha$ vs. $\mathrm{L} \beta$ ont été suffisamment en contact pour que des calques sémantiques soient plausibles. C'est le cas par exemple entre le moyen français et le moyen anglais à partir de la fin du $11^{\mathrm{e}}$ siècle, une fois le royaume d'Angleterre rattaché au duché de Normandie.

5. Gyrare (emprunt au grec үupeıv) est la source du fr. virer (qui s'est spécialisé dans le sens d'un mouvement rotatif dans la dimension horizontale) et des formations savantes giratoire et gyroscope (à partir du grec), vertere celle du fr. verser, (spécialisé dans le sens d'un mouvement rotatif dans la dimension verticale), torquere, par son dérivé extorquere est la source du fr. extorquer, volvere, celle de l'esp. volver et par son dérivé euoluere celle du fr.évoluer.

6. Le verbe traduisant généralement tourner en italien est girare, verbe lui aussi réversible. Tornare traduit les emplois intransitifs de retourner, en concurrence avec ritornare.

7. Cette observation dérive du principe de structuration combinatoire des prédications verbales en termes de caractères aspectuel et participatif que j'ai développé dans François (1989: chapitres 4 et $5 ; 1999 ; 2003$ : chapitres 2 et 3$)$.

8. De même que je ne traiterai pas le champ sémasiologique des dérivés fr. contourner détourner et retourner, et it. ritornare, je ferai également abstraction des emplois de to turn en composition avec une particule (to turn away, back, down, etc.). 
9. Cette étude est originellement issue d'un article de K. Baldinger paru en 1966 dans les Cahiers de lexicologie.

10. On suppose que dans le champ de la propriété $A$, le sémème est affecté (en fonction du contexte) d'une des deux valeurs A1 OU A2.

11. cf. site http://www.eva.mpg.de/lingua/conference/07-SemanticMaps/files/theme.html

12. Respectivement colexification et isolectal set dans l'original.

13. Je remercie Achim Stein de m'avoir aimablement donné accès à sa base de données lexicales Italienische Verben.

14. cf. Cavalli-Sforza (1997), en particulier le double arbre de classement des peuples et des langues p. 46-7. Du fait de l'adoption fréquente de la langue du conquérant, les relations entre les deux ensembles sont loin d'être isomorphes. Ainsi l'éthiopien et le berbère sont directement apparentés du point de vue de la généalogie linguistique (groupe afro-asiatique) et sont très éloignés du point de vue de la génétique des populations (hyper-groupes africain vs. asiatique).

15. Voir ma critique (François 2008) du rang hiérarchique élevé du trait de causativité dans le classement de Stein qui conduit à éloigner artificiellement des acceptions qui ne diffèrent que par ce trait.

16. G: Dictionnaire latin-français de F. Gaffiot; LVF : Les verbes français (base de données de J. Dubois \& F. Dubois-Charlier accessible sur le site du laboratoire MoDyCo, université Paris-Ouest) ; OED : Oxford English Dictionary (complété par l'étude de C. Paulin, 2010) ; WIV : Wörterbuch der italienischen Verben de P. Blumenthal \& G. Rovere, 1998) ; IV : Italienische Verben, base de données lexicale de A. Stein (cf. 2005).

17. Les représentations graphiques des figures 7 à 11 ont été élaborées à l'aide du logiciel de production de diagrammes X-Mind librement accessible sur la toile à l'URL http:// www.xmind.net/. Qu'il me soit permis une digression sur un point que je crois important, tout comme Benoit Habert. Si la linguistique doit devenir une science à part entière comme le réclame Gilbert Lazard (cf. Lazard 2009), elle ne peut pas se passer d'outils. Pour prendre un exemple extérieur à mon propos, la recherche sur les localisations cérébrales des fonctions cognitives, après avoir connu un début fulgurant avec les travaux pionniers de $\mathrm{P}$. Broca et $\mathrm{C}$. Wernicke (entre autres) dans la deuxième moitié $\mathrm{du} 19^{\mathrm{e}}$ siècle, n'a pas pu progresser au cours du $20^{\mathrm{e}}$ siècle parce que l'observation du cerveau des patients cérébrolésés, en particulier celui des aphasiques pour ce qui concerne la neuropsycholinguistique (cf. Nespoulous 2005), supposait sa dissection. Par ailleurs l'expérience de la sensation des membres amputés chez les invalides de guerre de la 1 ère guerre mondiale avait mis en doute la notion même de localisation cérébrale (cf. Goldstein 1931, Merleau-Ponty 1942). Il a fallu la découverte technique de la tomographie clinique et fonctionnelle par émission de positron (T.E.P.) puis de la tomographie par résonnance magnétique pour que les neurosciences puissent reprendre cette question à nouveaux frais. On peut en dire autant du développement de la linguistique des grands corpus des langues contemporaines qui renouvelle la réflexion sur la grammaticalité et l'acceptabilité en contexte, d'expressions qui se révèlent par ailleurs plus figées qu'on ne le croyait. Ce que permet le logiciel $\mathrm{X}$-Mind, c'est d'élaborer des diagrammes de différents types dont tous les embranchements peuvent être visualisés ou cachés. Cette propriété permet de composer un arbre sémasiologique complet d'une taille presque indifférente, puis de visualiser les seuls embranchements pertinents d'un point de vue ou d'un autre, par exemple seulement ceux qui conduisent à une feuille où figure l'un des trois verbes tourner (figure 9) to turn (figure 10) ou tornare (figure 11). Certes, ce n'est rien d'autre qu'une commodité technique, mais cela permet au linguiste de mieux se représenter les sous-espaces sémantiques partagés par les sous-arbres sémasiologiques particuliers de chacun des trois verbes

18. On remarque que la réflexivité sémantique passe par une construction réfléchie en français et en italien et par une construction intransitive en anglais, d'où l'intérêt d'opérer dans l'arbre sémasiologique général un classement sémantique et non syntaxique. 
19. L'article turn (v.) du Online Etymology Dictionary indique: "late O.E. turnian "to rotate, revolve" in part also from O.Fr. torner "to turn," both from L. tornare "turn on a lathe," from tornus "lathe," from Gk. tornos "lathe, tool for drawing circles," from PIE base *ter- "to rub, rub by turning, turn, twist" (...). Expression to turn (something) into (something else) probably retains the classical sense of "to shape on a lathe" (attested in Eng. from c.1300)."

\title{
RÉSUMÉS
}

Cet article poursuit un objectif méthodologique, la présentation comparée de trois modes de représentation de la polysémie d'un item : grille, carte et arbre sémasioogique et son illustration autour d'un phylum étymologique, celui du verbe latin tornare qui est à la source, entre autres des verbes français tourner, italien tornare et anglais to turn et de leurs dérivés et composés. Dans l'éventail des sens et des constructions des trois verbes, tourner occupe une position intermédiaire, mais se révèle plus proche de to turn que de l'italien tornare. A partir du sens unique du verbe latin ("fabriquer sur un tour de pottier») chacun des trois verbes à configuré son espace sémantique en fonction de l'ensemble de ses concurrents, y compris les verbes morphologiquement apparentés.

This article aims at comparing three various modes or representation of the polysemy of a lexical item : a semasiological grid, map or tree. The choice of the semasiological tree is illustrated with three verbs belonging to an etymological phylum, that of lat. tornare ("to design soth on a potter's lathe"), Fr. tourner, It. tornare and Engl. to turn as well as their derivatives and compounds. Originating in the unique meaning of lat. tornare, each of the three verbs built up its semantic space according to the set of its competitors including the morphologically related verbs.

\section{INDEX}

Mots-clés : polysémie verbale, champ onomasiologique et sémasiologique, lexicologie contrastive, actance, aspect

Keywords : verb polysemy, onomasiological / semasiological field, contrastive lexicology, actancy

\author{
AUTEUR \\ JACQUES FRANÇOIS \\ Université de Caen \& CRISCO, EA 4255 \\ jacques.francois@unicaen.fr \\ www.interlingua.fr
}

